\title{
Die häufigsten medizinischen Notfälle in der Zahnarztpraxis - Teil 5: Immunologische und endokrinologische Notfälle
}

Studien zeigen, dass ca. die Hälfte aller Zahnärzte in einem gegebenen Jahr mit einem medizinischen Notfall konfrontiert wird. Die allermeisten Zwischenfälle verlaufen glimpflich, aber ein geringer Teil dieser Notfälle sind ernsthaft. Zu diesen Notfällen zählt z. B. auch die akute Unterzuckerung bei Diabetikern.

\section{Diabetes mellitus}

Glukose ist die Hauptenergiequelle für alle Zellen im menschlichen Organismus und der einzige Energiespender, den das Gehirn verwerten kann. Ein zu hoher (Hyperglykämie) oder zu niedriger (Hypoglykämie) Blutzuckerspiegel führt zu unterschiedlichen Dysfunktionen des zentralen Nervensystems. Die homöostatischen Mechanismen sind darauf ausgerichtet, einen Blutzuckerspiegel von etwa $50 \mathrm{mg} / \mathrm{dl}$ aufrechtzuerhalten, wobei individuelle Schwankungen physiologisch sind. Der mittlere Blutzuckerspiegel bei normalen Menschen, die über Nacht fasten, liegt bei $92-100 \mathrm{mg} / \mathrm{dl}$, wobei die Bandbreite von $78-115 \mathrm{mg} / \mathrm{dl}$ noch als im Normbereich gilt. Der minimale Blutzuckerspiegel, den das Gehirn benötigt, um eine normale zerebrale Funktion aufrechtzuerhalten, liegt bei $50 \mathrm{mg} / \mathrm{dl}$. Sobald der Blutzuckerspiegel den Sättigungspunkt der Nierenreabsorption (etwa $180 \mathrm{mg} / \mathrm{dl}$ ) übersteigt, kommt es zum „Überlaufen“ von Glukose in den Urin, was zu einem Verlust von Glukose und Wasser führt.

Insulin ist der wichtigste Faktor bei der Regulierung des Blutzuckerspiegels. Es wird in den Betazellen des Pankreas synthetisiert und als Reaktion auf Erhöhungen des Blutzuckerspiegels (z.B. nach einer Mahlzeit) rasch ins Blut abgesondert. Insulin wird hepatisch und renal metabolisiert. Endogen synthetisiertes Insulin weist eine kurze Halbwertszeit von 3-5 min auf, die Metabolisierung erfolgt zu $40-50 \%$ in der Leber und zu 30$80 \%$ in den Nieren $[1,2]$. Insulin fördert die Aufnahme von Glukose in die Körperzellen und dessen Lagerung in der Leber als Glykogen. Auch unterstützt es die Aufnahme von Fettsäuren und Aminosäuren in die Zellen und ihre anschließende Umwandlung zu Speicherungsformen (Triglyceride und Proteine). Auf diese Weise führt Insulin zu einem Rückgang des Blutzuckerspiegels und verhindert, dass es durch Urinausscheidung verloren geht. Ohne Insulin ist die Membran vieler Zellen nicht glukosepermeabel. Muskeln und Fettzellen sind insulinabhängig, damit Glukose die Zellmembran durchqueren kann. Fehlt Insulin, spalten diese Zellen Triglyceride zu Fettsäuren auf, die der Körper als alternative Energiequelle nutzen kann. Dieser Prozess ruft einen hyperglykämischen Zustand hervor, der als diabetische Ketoazidose bekannt ist. Andere Gewebe und Organe, wie das Nervengewebe (einschließlich Gehirn), die Nieren und Lebergewebe, sind nicht insulinabhängig. Diese Gewebe können Glukose auch ohne Insulin durch die Zellmembran transferieren.

Niedrige Blutzuckerspiegel hemmen die Insulinsekretion, wobei die Körperzellen weiterhin Glukose verwerten müssen. Es gibt verschiedene Mechanismen, um dies zu erreichen. Ziel ist v.a., das zentrale Nervensystem mit einem Mindestspiegel an Glukose zu versorgen, damit es seine Funktion aufrechterhalten kann. Glykogen wird in der Leber gespalten, und die Speicher werden dort abgebaut - ein Prozess, der Glykogenolyse genannt wird. Die Glukoneogenese beschreibt die Umwandlung von Proteinbausteinen, den Aminosäuren, zu Glukose. Während diese Prozesse in der Lage sind, dem ZNS eine ausreichende Versorgung mit Glukose zu ermöglichen, werden andere insulinabhängige Zellen minderversorgt. Energiespender für diese Zellen (z. B. Muskel- und Fettzellen) werden durch die Aufspaltung von Triglyceriden, der Speicherungsform von Fett, in freie Fettsäuren geliefert.

\section{Klassifizierung von Diabetes}

Bis vor kurzem basierte die Klassifizierung eines Diabetes auf dem Alter bei Ausbruch der Krank- 
heit (Altersdiabetes und juveniler Diabetes), und ob zur Behandlung der Erkrankung Insulininjektionen erforderlich waren oder nicht (insulinabhängiger Diabetes mellitus und nicht insulinabhängiger Diabetes mellitus). Die Klassifikation hat sich verfeinert, und so ist Diabetes mellitus nun der Sammelbegriff für heterogene Störungen des Stoffwechsels, deren Leitbefund die chronische Hyperglykämie ist. Ursache ist entweder eine gestörte Insulinsekretion oder eine gestörte Insulinwirkung oder auch beides ( 0 Tab. 1, 2).

Typ-1-Diabetes:

- Betazellzerstörung, die zu einem absoluten Insulinmangel führt

- meist immunologisch vermittelt

- Der LADA (latent autoimmune diabetes in adults) wird dem Typ-1 zugeordnet.

Typ-2-Diabetes:

- kann sich erstrecken von einer vorwiegenden Insulinresistenz mit relativem Insulinmangel bis zu einem vorwiegend sekretorischen Defekt mit Insulinresistenz

- ist häufig assoziiert mit anderen Problemen eines sog. metabolischen Syndroms

Andere spezifische Diabetestypen:

- Erkrankungen des exokrinen Pankreas (z.B. Pankreatitis, zystische Fibrose, Hämochromatose)

- Endokrinopathien (z.B. Cushing-Syndrom, Akromegalie, Phäochromozytom)

- medikamentös-chemisch induziert (z.B. Glukokortikoide, Neuroleptika, Alpha-Interferon, Pentamidin)
- genetische Defekte der Betazellfunktion (z.B. MODY-Formen)

- genetische Defekte der Insulinwirkung

\section{Hypoglykämie}

Es gibt in der Zahnarztpraxis 2 mögliche Komplikationen im Zusammenhang mit einem Diabetes mellitus: die Hyperglykämie oder ein zu hoher Blutzuckerspiegel und die Hypoglykämie oder der zu niedrige Blutzucker. Lebensbedrohlich ist die Hypoglykämie, sodass wir hier weitgehend auf diesen endokrinologischen Notfall näher eingehen wollen.

Zur Diagnose von Diabetes werden folgende Blutzuckerwerte herangezogen: Gelegenheits-Plasmaglukosewert von $\geq 200 \mathrm{mg} / \mathrm{dl}(\geq 11,1 \mathrm{mmol} / \mathrm{l})$ oder Nüchternplasmaglukose von $\geq 126 \mathrm{mg} / \mathrm{dl}$ ( $\geq 7,0 \mathrm{mmol} / \mathrm{l})$. Eine Hypoglykämie kann sowohl bei Diabetikern als auch bei Nichtdiabetikern auftreten. Blutzuckerspiegel unter $70 \mathrm{mg}$ pro $100 \mathrm{ml}$ $(\mathrm{mg} / \mathrm{dl})$ stellen bei Erwachsenen und pädiatrischen Diabetikern eine Hypoglykämie dar [3].

Eine Hypoglykämie kann sich rasch entwickeln, innerhalb von Minuten bei insulinpfichtigen Diabetikern und innerhalb von Stunden bei Patienten, die orale Antidiabetika einnehmen. Je nach Ausprägung der Unterzuckerung kann es zur Bewusstlosigkeit und einer vitalen Gefährdung kommen ( $\bullet$ Tab. 3). In dem Fall wäre eine orale Gabe von Glukose wegen der Aspirationsgefahr kontraindiziert.

Faktoren, die den Insulinbedarf des Körpers senken (Hypoglykämie):

\begin{tabular}{l|l|}
\hline $\begin{array}{l}\text { Tab. } 1 \text { Klassifikation der Diabetestypen. } \\
\text { Diabetes mellitus }\end{array}$ & $\begin{array}{l}\text { Typ } 1 \text { (früher insulinabhängiger oder juveniler Diabetes) immunologisch vermittelt idiopathisch } \\
\text { Typ } 2 \text { (früher nicht insulinabhängiger oder Altersdiabetes) }\end{array}$ \\
\hline $\begin{array}{l}\text { weitere spezifische } \\
\text { Formen }\end{array}$ & $\begin{array}{l}\text { genetische Defekte der Betazellfunktion } \\
\text { genetische Defekte der Insulinaktivität } \\
\text { Erkrankungen des exokrinen Pankreas } \\
\text { medikamenten- oder chemikalieninduzierte Infektionen } \\
\text { ungewöhnliche Formen von immunologisch vermitteltem Diabetes }\end{array}$ \\
\hline $\begin{array}{l}\text { weitere genetische } \\
\text { Syndrome }\end{array}$ & $\begin{array}{l}\text { Schwangerschaftsdiabetes } \\
\text { beeinträchtigte Glukosetoleranz } \\
\text { gestörte Nüchternglukose }\end{array}$ \\
\hline
\end{tabular}

Tab. 2 Vergleich zwischen Type-1 und Typ-2-Diabetes.

\begin{tabular}{|l|l|l|}
\hline Faktor & Typ $\mathbf{1}$ & Typ 2 \\
\hline Häufigkeit (in \% der gesamten diabetischen Bevölkerung) & 10 & 90 \\
\hline Alter bei Ausbruch [Jahre] & 15 & $\geq 40$ \\
\hline Körperbau & normal & oft übergewichtig \\
\hline Schweregrad & schwer & mittel \\
\hline Einsatz von Insulin & fast alle & $20-30 \%$ \\
\hline Reaktion auf orale blutzuckersenkende Mittel & kaum & $50 \%$ \\
\hline Ketoazidose & häufig & selten \\
\hline Komplikationen & $90 \%$ in 2 Jahren & weniger akut \\
\hline Geschwindigkeit des klinischen Ausbruchs & rasch & langsam \\
\hline Stabilität & instabil & stabil \\
\hline Familienanamnese & häufig & weniger häufig als Typ1 \\
\hline humane Lymphozytenantigene und anomale Autoimmunreaktion & liegen vor & liegen nicht vor \\
\hline Defekte der Insulinrezeptoren & Nein & Ja \\
\hline
\end{tabular}


- Untergewicht

- körperliche Bewegung

- Pharmaka (Schilddrüsenhormone, Kortikoide)

- Auslassen oder Verspätung von Mahlzeiten

- intensive körperliche Anstrengung vor dem Essen oder erhöhter Insulindosis

- ohne jeden ersichtlichen Grund

Eine Zahnbehandlung kann für Diabetiker risikoreich sein. Stress erhöht den Bedarf des Körpers

\begin{tabular}{c|l}
\hline \multicolumn{1}{l}{$\begin{array}{l}\text { Tab. } 3 \text { Klinische Zeichen einer Hypoglykämie. } \\
\text { frühes Stadium (leichte Reaktionene) }\end{array}$} & Tremor \\
& Stimmungsschwankungen \\
& Hunger \\
& Übelkeit \\
\hline schweres frühes Stadium & Schwitzen \\
& Tachykardie \\
& Angstgefühle \\
& bizarre Verhaltensmuster, ZNS-Dysfunktion \\
& mangelnde Urteilsfähigkeit \\
& unkooperativ \\
\hline späteres schweres Stadium & Bewusstlosigkeit \\
& Krampfaktivität \\
& Hypotonie \\
& Hypothermie \\
\hline
\end{tabular}

Problem erkennen (veränderter Bewusstseinszustand)

Zahnbehandlung einstellen

Notfallteam der Praxis aktivieren

$\mathbf{P}$ - Patienten bequem positionieren

A > B > C Atemwege freimachen, Atmung prüfen, Karotispuls max. 10 s prüfen Falls erforderlich, lebensrettende Sofortmaßnahmen (BLS) durchführen

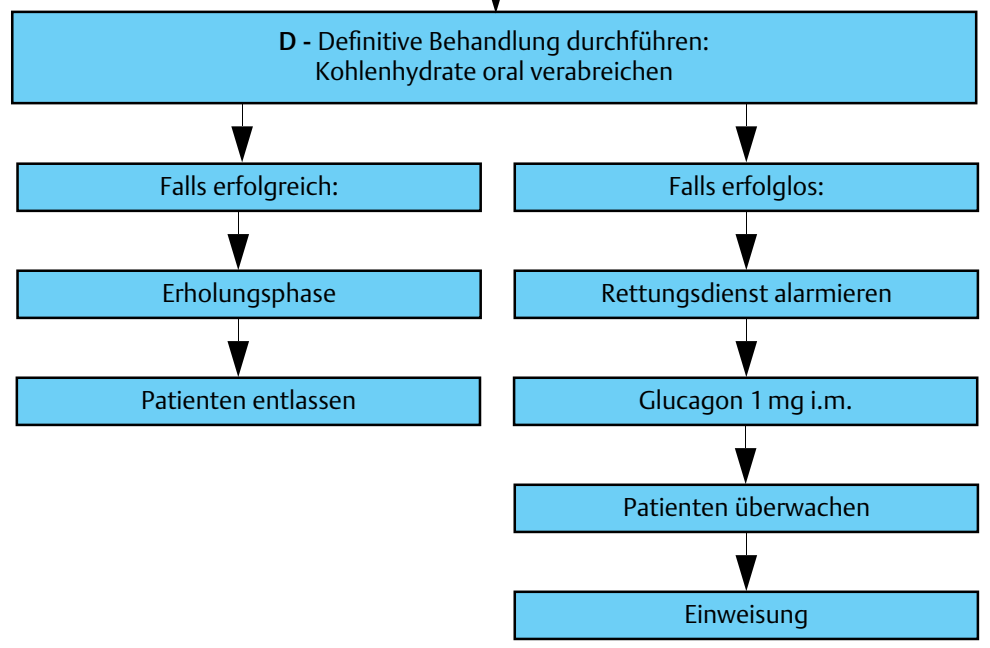

Abb. 1 Behandlung einer Hypoglykämie - Patient bei Bewusstsein. an Insulin, was das Risiko des Patienten, eine Hyperglykämie zu entwickeln, steigert. Hinzu kommt, dass die Zahnbehandlung Veränderungen der normalen Essgewohnheiten des Patienten bedeuten und dies wiederum mit einer Hypoglykämie einhergehen kann.

\section{Behandlung}

Die Behandlung von Hypoglykämie liefert weitaus dramatischere Ergebnisse als die der Hyperglykämie; bei den meisten Patienten tritt eine sofortige Besserung der Symptome innerhalb kürzester Zeit ein. Die Behandlungsstrategie hängt vom Bewusstseinsstadium des Patienten ab.

Die folgenden Zeichen stellen diagnostische Anhaltspunkte über das Vorliegen einer Hypoglykämie dar:

- Schwäche

- Verwirrtheit

- blasse, feuchte Haut

- normale oder gedämpfte Atemzüge

- Kopfschmerzen

- veränderter Bewusstseinszustand

Bizarres Verhalten, Persönlichkeitsveränderungen und andere klinische Zeichen der Hypoglykämie treten meist bei bekannten Diabetikern auf. Nichtdiabetiker hingegen zeigen diese Symptome nur sehr selten. Bei Diabetikern sollte der Arzt ggf. bei Angehörigen in Erfahrung bringen, wann die letzten Nahrungsaufnahme und Insulindosis bei dem Patienten erfolgten.

Wenn der Patient bei Bewusstsein und kooperativ ist, aber noch immer klinische Zeichen einer Hypoglykämie zeigt, sind orale Kohlenhydrate das Mittel der Wahl. Das Notfallset der Zahnarztpraxis enthält Glukose, das vom Patienten zu sich genommen werden kann. Andere mögliche Quellen können Orangensaft und Softdrinks sein. Eine 170- bzw. 340-g-Portion Softdrink enthält 20-40g Glukose. Die Kohlenhydrate sollten in 3-4 Portionen von jeweils etwa $100 \mathrm{~g}$ alle $5-10 \mathrm{~min}$ gegeben werden, bis die Symptome verschwinden.

Bei bewusstlosen Patienten mit Zeichen und Symptomen einer Hypoglykämie werden keine oralen Kohlenhydrate verabreicht, da die Patienten keine Schutzreflexe haben und eine Aspiration droht. Hier muss entweder Glukose parenteral gegeben werden oder $1 \mathrm{mg}$ Glukagon intramuskulär oder subkutan gespritzt werden. Im Notfallset der Zahnarztpraxis befindet sich Glucagon zur intramuskulären/subkutanen Applikation bei der Hypoglykämie, und die meisten Zahnärzte werden diese Notfallbehandlung wählen. Die Gabe von Glukagon $1 \mathrm{mg}$ intramuskulär/ subkutan führt zu einer Erhöhung des Blutzuckers über eine Aufspaltung der Glykogendepots in der Leber. Die Reaktion von Glukagon variiert, wobei ein Einsetzen der Wirkung zwischen 10 und 20 min dauert und ein Erreichen des Spitzenwertes innerhalb von 30-60 min erfolgt. Steht kein Glukagon zur Verfügung, können 0,3 mg Adrenalin (Fastjekt) subkutan gegeben und dies bei 
Bedarf nach 15 min wiederholt werden. Adrenalin erhöht den Blutzuckerspiegel, sollte aber bei Patienten mit bekannter kardiovaskulärer Erkrankung mit Vorsicht eingesetzt werden ( $\bullet$ Abb. 1). Der bewusstlose hypoglykämische Patient erlangt das Bewusstsein, sobald der Blutzuckerspiegel erhöht wird und solange keine zusätzlichen Schädigungen (z.B. durch Hypoxie) eingetreten sind. Ist der Patient bei Bewusstsein, können ihm orale Kohlenhydrate, wie Fruchtsaft oder Softdrinks, gegeben werden. Bei seinem Eintreffen sichert der Rettungsdienst die lebensrettenden Sofortmaßnahmen, legt einen intravenösen Zugang und transportiert den Patienten zur definitiven Versorgung und Beobachtung ins Krankenhaus ( $\bullet$ Abb. 2).

\section{Anaphylaxie}

Die Anaphylaxie ist eine lebensbedrohliche fulminante immunologische Reaktion. Nach Kontakt mit dem Allergen werden Entzündungsmediatoren freigesetzt (Histamin, Prostaglandine, Thromboxane, Plättchen-Wachstumsfaktoren (PDGF) und Leukotrien). Klinisch kann es zum kardiovaskulären Kollaps und respiratorischer Insuffizienz kommen. Eine frühzeitige Behandlung mit intramuskulärem Adrenalin, einem Alpharezeptor-Agonist, führt zur Stabilisierung der Vasodilatation. Die Aktivierung der Betarezeptoren bedingt eine Weitstellung der Bronchien und eine Steigerung der myokardialen Kontraktilität. Die Freisetzung von Histamin und Leukotrien wird unterdrückt.

\section{Auslöser einer Anaphylaxie}

Antibiotika (Penicilline, Sulfonamide)

Parenteral verabreichtes Penicillin kann eine anaphylaktische Reaktion hervorrufen. Eine orale Gabe verursacht normalerweise eine verzögerte Reaktion. Patienten wissen möglicherweise nicht, dass sie zuvor bereits einer Sensibilisierungsdosis ausgesetzt waren (Fleisch, Milch).

Analgetika (Aspirin, Codein, NSAR)

Die Symptome reichen von leichter Urtikaria bis hin zur Anaphylaxie. Die häufigste Reaktion besteht in einem Bronchospasmus. Bei Patienten mit bekannter Allergie auf die o.g. Analgetika sollten Alternativen (z. B. Paracetamol) verschrieben werden.

Lokalanästhetika (Ester, Procain, Benzocain) Injizierbare und topische Lokalanästhetika vom Estertyp waren vorrangig in allergische Reaktionen verwickelt. Allergische Reaktionen, die bei Amiden berichtet wurden, sind möglicherweise durch Konservierungsmittel, wie Parabene, verursacht.

\section{Symptome der Anaphylaxie}

Haut:

- Urtikaria, Juckreiz, Nesselsucht

- Erythem
- Angioödem, lokalisierte Schwellung

Atemwege:

- Bronchospasmus, Atemnot, Keuchen

- Angioödem des Kehlkopfes

- Rhinitis

Kardiovaskulär:

- Kreislaufinsuffizienz durch Vasodilatation

- Herzrhythmusstörungen bis zur Asystolie

Behandlung

Patienten, die Anzeichen einer Anaphylaxie zeigen, sollten 61/min Sauerstoff per Nasensonde oder Maske erhalten. Überwachung der Vitalzeichen: Die Vitalzeichen einschließlich Blutdruck, Herzfrequenz und -rhythmus sowie die Atemfrequenz sollten alle $5 \mathrm{~min}$ gemessen und dokumentiert werden. Pharmakotherapie: Der Jext/ Fastjekt junior ist speziell für Kinder von 1-8 Jahren (15-30 kg KG) konzipiert und erhält $0,15 \mathrm{mg}$ Adrenalin in $0,3 \mathrm{ml}$ Injektionslösung. Dies entspricht der empfohlenen Dosierung für die Behandlung einer allergischen Reaktion. Die Injektion erfolgt intramuskulär am lateralen Oberschenkel und wird alle 5 min wiederholt, bis sich die Symptomatik gebessert hat. Kindern mit mehr als $30 \mathrm{~kg}$ Körpergewicht und Erwachsene erhalten Jext/Fastjekt für Erwachsene mit $0,3 \mathrm{mg}$ Adrenalin pro Einmaldosis. Zur erweiterten Behandlung einer anaphylaktischen Reaktion kann nach der Stabilisierung des Patienten Adrenalin, Hydrocortison gegeben werden. Kortikoide gehören nicht zur Primärbehandlung anaphylakti-

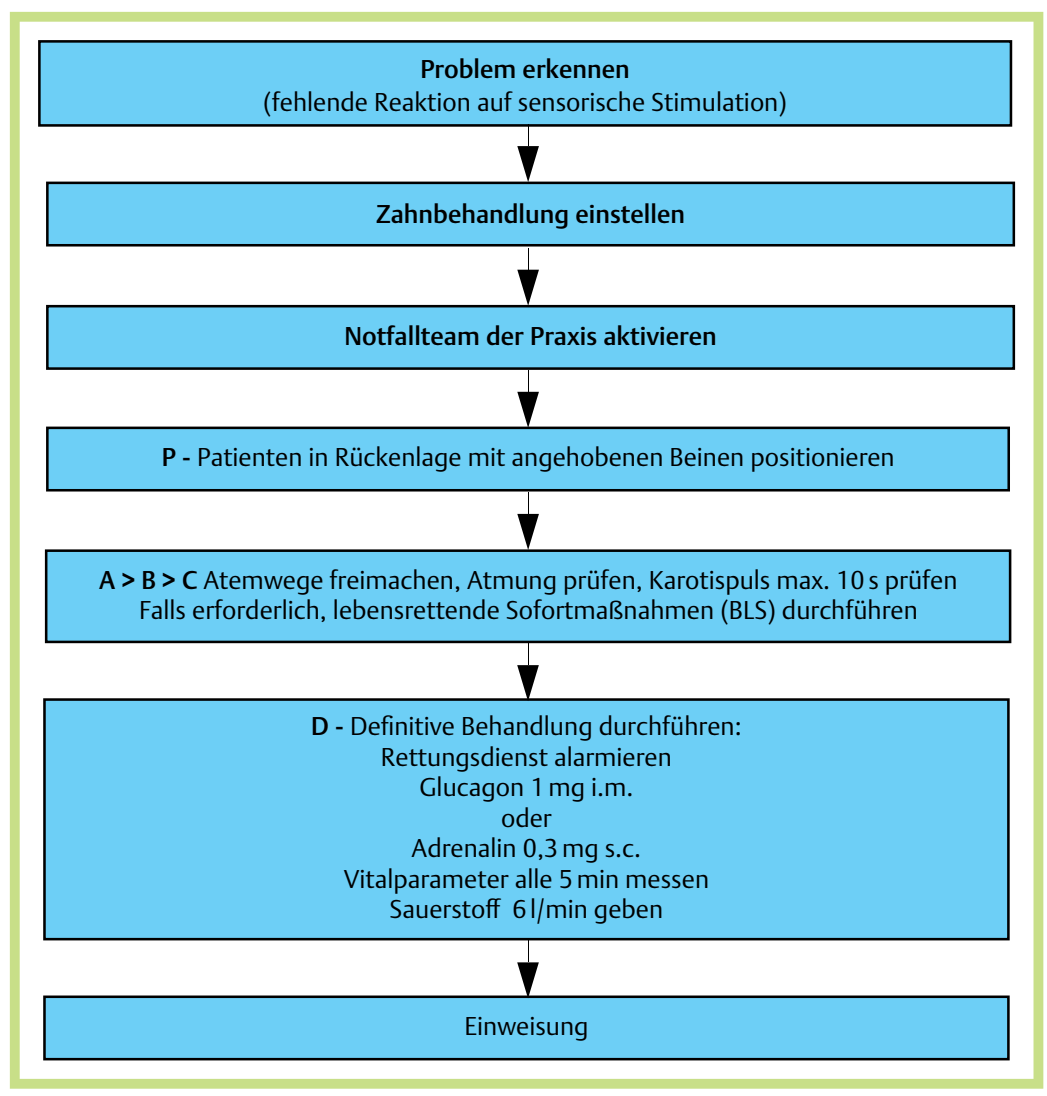

Abb. 2 Behandlung einer Hypoglykämie - Patient bewusstlos. 


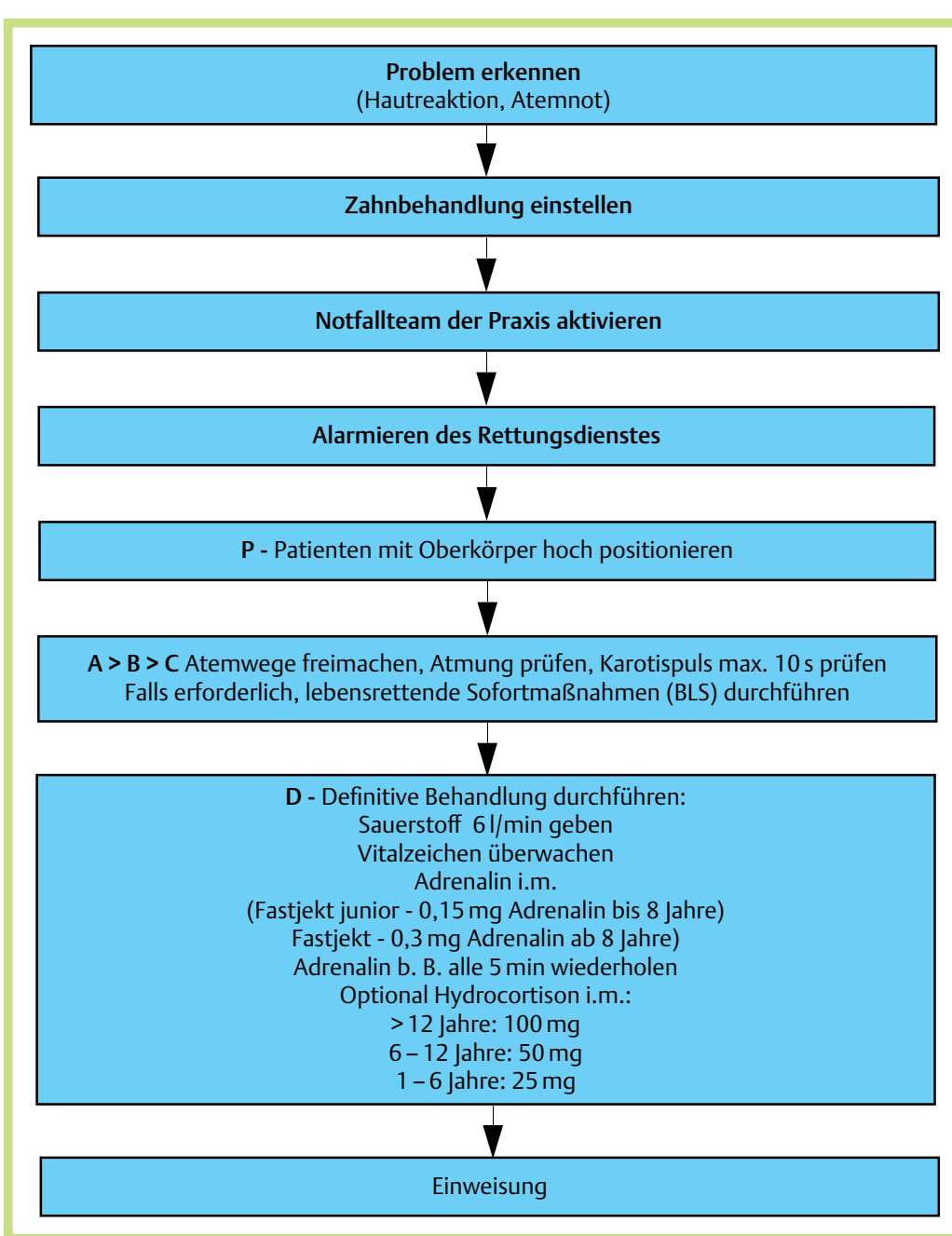

Abb. 3 Behandlung einer Anaphylaxie.

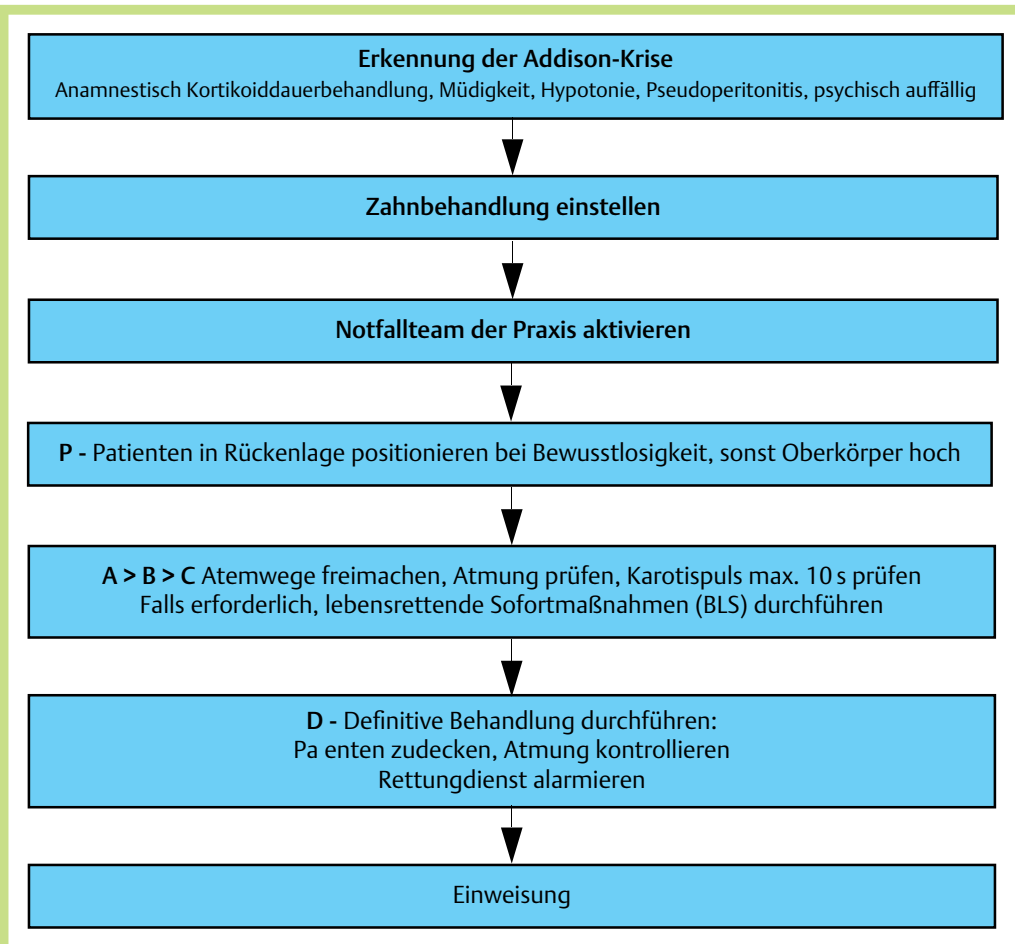

Abb. 4 Behandlung einer Addisonkrise. scher Reaktionen, aber sie hemmen die Freisetzung von Histamin und können die Symptomatik des anaphylaktischen Schocks und schwerer allergischer Reaktionen mildern. Der größte Nachteil beim Einsatz von Kortikosteroiden in Notfallsituationen besteht in dem langsamen Einsetzen der Wirkung (mehr als $1 \mathrm{~h}$ ). Aus diesem Grund sind sie nicht als unabdingbarer Bestandteil der Notfallausrüstung anzusehen ( $\bullet$ Abb. 3).

Hydrocortison wird, sofern kein intravenöser Zugang vorhanden ist, intramuskulär injiziert.

Dosierung von Hydrocortison i.m.:

- 1-6 Jahre $25 \mathrm{mg}$ i.m. oder i.v.

- 6-12 Jahre $50 \mathrm{mg}$ i.m. oder i.v.

- >12 Jahre und Erwachsene $100 \mathrm{mg}$ i.m. oder i.v.

Patienten, die eine Anaphylaxie in der Zahnarztpraxis entwickeln, müssen nach der Primärversorgung mit Notarztbegleitung in die Klinik transportiert werden.

\section{Addisonkrise}

Die Nebennierenrinde produziert 3 Steroidhormone: Glukokortikoide (Cortisol), Mineralokortikoide und Androgene. Cortisol ist ein lebenswichtiges Glukokortikoid, denn es reguliert wichtige metabolische, kardiovaskuläre, immunologische und homöostatische Funktionen. Eine akute Insuffizienz der Nebenniere (Addisonkrise) kann durch den Stress eines zahnärztlichen Eingriffs bei bestehender chronischer Nebenniereninsuffizienz ausgelöst werden. Selten wird eine primäre Nebenniereninsuffizienz durch eine Degeneration der Nebennieren (z.B. Tbc) oder autoimmun verursacht. Vielmehr findet sich meist eine sekundäre Nebenniereninsuffizienz infolge einer hypothalamisch-hypophysären Dysfunktion als Folge einer iatrogenen exogenen Steroidzufuhr. Die Cortisolproduktion wird normalerweise als Reaktion auf Stress erhöht; wenn jedoch die Nebennierenrinde nicht in der Lage ist, eine Cortisolmenge zu produzieren, die für den erhöhten Bedarf ausreicht, kann es zu einer Addisonkrise, einem potenziell lebensbedrohlichen Notfall kommen.

Risikopatienten sollten Termine am frühen Morgen bekommen, da der endogene Cortisolspiegel dann am höchsten ist. Es muss sichergestellt sein, dass Patienten ihre übliche morgendliche Steroiddosis vor dem Eingriff eingenommen haben. Bei Bedarf sollten Medikamente gegen Schmerzen und Angst gegeben werden.

Klinische Zeichen und Symptome einer AddisonKrise:

- Müdigkeit, Lethargie

- Hyperpigmentierung der Haut und Schleimhäute

- Muskelschwäche

- Kopfschmerzen, Verwirrung, Fieber

- Übelkeit, Erbrechen, Bauchschmerzen

- Hypotonie, Tachykardie

- Schwitzen und Dehydrierung 
Eine Hypotonie ohne angemessene Glukokortikoidspiegel im Blut kann bei entsprechender Belastung (Stress, medizinische Behandlung usw.) zu einer akuten Herz-Kreislauf-Insuffizienz führen. Ohne Behandlung kann es zum letalen Ausgang führen.

\section{Behandlung}

Die Behandlung einer Nebenniereninsuffizienz erfordert nur eine Stabilisierung der lebenswichtigen Funktionen bis zur Übergabe an den Rettungsdienst. Die Atemwege müssen gesichert und die Vitalparameter kontinuierlich überwacht werden. Kommt es zur Dyspnoe, sollte Sauerstoff mit 61/min über eine Nasenkanüle gegeben werden. Diese Patienten können präklinisch nicht umfassend behandelt werden, benötigen eine intensivmedizinische Betreuung u.a. mit der Gabe von Volumenersatz, Glukokortikoiden und ggf. Glukose intravenös, um eine vorliegende Hypoglykämie zu behandeln. Sekundäre Komplikationen, wie Herzrhythmusstörungen aufgrund von Elektrolytentgleisungen, Pseudoperitonitis, Exsikkose und Schock, sind weitere bekannte Komplikationen einer Addison-Krise, die sich einer präklinischen Behandlung weitgehend entziehen (๑ Abb. 4).
Prävention einer Addison-Krise

Das Risiko einer Addison-Krise besteht bis zu 12 Monaten nach dem Absetzen von Kortikosteroiden. Stress, einschließlich einer Zahnbehandlung, kann eine Addison-Krise auslösen, sodass eine enge Abstimmung zwischen Zahnarzt und dem Hausarzt/der Hausärztin stattfinden muss. Es sollte erwogen werden, u.U. die Kortikosteroidtherapie im Rahmen einer größeren zahnmedizinischen Behandlung wieder aufzunehmen oder eine laufende Dosis zu erhöhen [4]. Viele Zahnärzte werden bei diesen Patienten eine dentale Sedierung mit Lachgas durchführen, um den Behandlungsstress zu minimieren. Eine ausreichende Analgesie für die Postbehandlungsphase trägt ebenfalls zu einem komplikationslosen Verlauf bei diesen Patienten bei.

Interessenkonflikt: Es liegt kein Interessenkonflikt vor.

\section{Literatur}

1 Rabkin R, Rayn MP, Duckworth WC. The renal metabolism of insulin. Diabetologia 1984; 27: 351-357

2 Mak RH, DeFronzo RA. Glucose and insulin metabolism in uremia. Nephron 1992; 61: 377-382

3 Seaquist ER, Anderson J, Childs B et al. Hypoglycemia and diabetes: a report of a workgroup of the American Diabetes Association and the Endocrine Society. Diabetes Care 2013; 36: 1384-1395

4 Henderson S. What steroid supplementation is required for a patient with primary adrenal insufficiency undergoing a dental procedure? Dent Update 2014; 41: 342-344
Bibliografie

DOI http://dx.doi.org/ 10.1055/s-0042-121330 ZWR - Das Deutsche Zahnärzteblatt 2016; 125: 628-633 (c) Georg Thieme Verlag KG Stuttgart · New York ISSN 0044-166X

Korrespondenzadresse Dr. med. Frank G. Mathers Institut für dentale Sedierung Goltsteinstraße 95 50968 Köln info@sedierung.com fgm4@yahoo.com 